\title{
Ginsenoside 20(S)-Rg3 Prevents PKM2- Targeting miR-324-5p from H19 Sponging to Antagonize the Warburg Effect in Ovarian Cancer Cells
}

\author{
Xia Zheng ${ }^{a, b}$ Yuanyuan Zhou ${ }^{a, b}$ Wei Chen ${ }^{c}$ Lihong Chen ${ }^{d}$ Jiaojiao Lu,b \\ Fang $\mathrm{He}^{\mathrm{a}, \mathrm{b}} \quad \mathrm{Xu} \mathrm{Li}^{\mathrm{a}} \mathrm{b} \quad$ Le Zhao ${ }^{\mathrm{a}, \mathrm{b}}$
}

${ }^{a}$ Center for Translational Medicine, the First Affiliated Hospital of Xi'an Jiaotong University, Xi'an, ${ }^{b}$ Key Laboratory for Tumor Precision Medicine of Shaanxi Province, the First Affiliated Hospital of Xi'an Jiaotong University, Xi'an, 'Center for Laboratory Medicine, the First Affiliated Hospital of Xi'an Jiaotong University, Xi'an, dDepartment of Obstetrics and Gynecology, Shaanxi Provincial People's Hospital, Xi'an, China

\section{Key Words}

Ginsenoside $\cdot$ Ovarian cancer $\cdot$ Warburg effect $•$ Long non-coding RNA

\begin{abstract}
Background/Aims: The Warburg effect is one of the main metabolic features for cancers, with long non-coding RNA (IncRNA) being involved as a class of crucial regulators. Our previous studies have shown that ginsenoside 20(S)-Rg3, an active saponin monomer extracted from red ginseng, inhibits the Warburg effect in ovarian cancer cells. However, the detailed IncRNA regulatory network modulated by $20(\mathrm{~S})$ - Rg3 to prevent the Warburg effect in ovarian cancer cells has not been explored. Methods: High-throughput sequencing was used to screen out the differentially expressed IncRNAs between 20(S)-Rg3-treated and non-treated SKOV3 cells. The levels of IncRNA H19 and miR-324-5p were manipulated in SKOV3 and A2780, and the glucose consumption, lactate production and PKM2 protein level were detected. Dualluciferase reporter assay and RIP were utilized to verify the direct binding of $\mathrm{H} 19$ to miR-324$5 p$ and miR-324-5p to PKM2. Cell proliferation was examined by CCK8 and colony formation assay. Nude mice subcutaneous xenograft tumor models were established to evaluate the impact of miR-324-5p on tumor growth in vivo. Results: $20(\mathrm{~S})-\mathrm{Rg} 3$ downregulated 67 IncRNAs, and $\mathrm{H} 19$ was one of the most decreased IncRNAs. Suppression of H19 by siRNA transfection reduced glucose consumption, lactate production and PKM2 expression in ovarian cancer cells, while H19 overexpression in 20(S)-Rg3-treated ovarian cancer cells enhanced glucose consumption, lactate production and PKM2 expression. Dual-luciferase reporter assay and RIP results showed that $\mathrm{H} 19$ directly bound to miR-324-5p. Dual-luciferase reporter assay showed that miR-324-5p directly targeted PKM2, and miR-324-5p negatively

$X$. Zheng and $Y$. Zhou contributed equally to this work.

Le Zhao, Ph.D

and Xu Li, Ph.D

Center for Translational Medicine, Key Laboratory for Tumor Precision Medicine of Shaanxi Province, the First Affiliated Hospital of Xi'an Jiaotong University, Xi'an, 710061 (China) Tel. +86-29-85323528, E-Mail zhaole2@mail.xjtu.edu.cn; lixu56@mail.xjtu.edu.cn
\end{abstract}

KARGER 


\section{Cellular Physiology Cell Physiol Biochem 2018;51:1340-1353

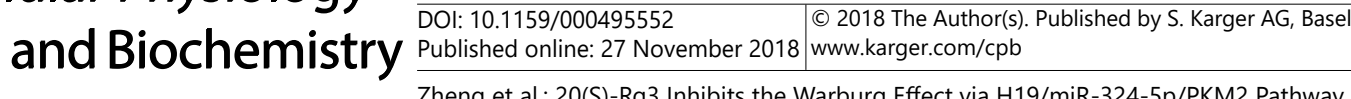

regulated glucose consumption and lactate production in ovarian cancer cells. miR-324-5p overexpression inhibited cell proliferation in vitro and in vivo. Conclusion: Our study revealed that 20 (S)-Rg3 blocked the competitive inhibition of H19 on miR-324-5p, which enhanced the suppression of miR-324-5p on PKM2 and therefore inhibited the Warburg effect and repressed tumorigenesis. In a word, 20(S)-Rg3 inhibited the Warburg effect in ovarian cancer cells via H19/miR-324-5p/PKM2 pathway.

(C) 2018 The Author(s)

Published by S. Karger AG, Basel

\section{Introduction}

Ovarian cancer is the most lethal gynecological tumor, existing predominantly in the form of epithelial ovarian cancer (EOC). The estimated new cases of ovarian cancer are 22,240 , and the estimated deaths are 14,070 , which is $5 \%$ of deaths of females in the United States [1]. The 5-year survival has long been less than $50 \%$ in most countries [2]. Chemotherapy is the main adjuvant therapy for EOC, however, its efficacy is frequently impaired by occurrence of chemoresistance and adverse reaction [3]. Development of novel therapeutics is an important strategy to improve the prognosis of EOC patients.

Ginsenoside 20(S)-Rg3 is an active saponin monomer extracted from red ginseng, exerting anti-tumor function in multiple cancers [4]. A series of study have shown that 20(S)Rg3 significantly inhibits ovarian cancer cells growth and metastasis partly via antagonizing the Warburg effect $[5,6]$. The Warburg effect, also termed as aerobic glycolysis, is the main metabolic feature of cancers sustaining cancer cell growth under sufficient oxygen via rapidly generating ATP and biomaterials [7]. The Warburg effect is controlled by key glycolytic enzymes including hexokinase (HK), phosphofructokinase (PFK), pyruvate kinase (PK), and lactate dehydrogenase (LDH) [8]. Lately, long non-coding RNAs have been identified as a class of crucial regulators of the Warburg effect $[9,10]$. However, the detailed lncRNA regulatory network modulated by $20(\mathrm{~S})-\mathrm{Rg} 3$ to prevent the Warburg effect in ovarian cancer cells has not been fully explored.

LncRNAs are a class of non-coding RNAs that are longer than 200 nucleotides implicated in tumor development and progression via regulating multiple genes expression to participate in various biological processes including metabolism, proliferation, apoptosis, migration and invasion. LncRNAs play their regulation role partly through acting as microRNA sponges to antagonize the binding of microRNA to their targets at 3' untranslated regions (3'UTRs) and therefore prevent microRNA-rendered promotion of RNA degradation or inhibition of translation. Increasing evidences have shown that lncRNAs regulate the Warburg effect in various cancers via sponging microRNAs $[11,12]$.

Here, we screen the differentially expressed lncRNAs between 20(S)-Rg3-treated and non-treated ovarian cancer cells via deep sequencing, and establish one IncRNA regulatory pathway modulated by $20(\mathrm{~S})-\mathrm{Rg} 3$ to prevent the Warburg effect in ovarian cancer cells. The results provide essential information for clinical translation of $20(\mathrm{~S})$-Rg3 for ovarian cancer therapy.

\section{Materials and Methods}

Drugs and antibodies

Ginsenoside 20(S)-Rg3 was obtained from Tasly Pharmaceutical Company (Tianjin, China). 20(S)Rg3 was dissolved in DMSO, and then diluted in RPMI 1640 media at the concentration of $2 \mathrm{mg} / \mathrm{ml}$ as a stock solution and stored at $-20^{\circ} \mathrm{C}$ for later use. The primary antibodies against PKM2 (\#4053) and $\beta$-actin (\#3700) were from Cell Signaling Technology (Beverly, MA, USA), and the primary antibody against Ago2 (03-110) was from Millipore (Billerica, MA, USA). 


\section{Cellular Physiology Cell Physiol Biochem 2018;51:1340-1353 \begin{tabular}{ll|l} 
and Biochemistry Published online: 27 November 2018 & $\begin{array}{l}\text { (c) } 2018 \text { The Author(s). Published by S. Karger AG, Basel } \\
\text { www.karger.com/cpb }\end{array}$ \\
\hline
\end{tabular}

\section{Cell culture and treatment}

The human ovarian cancer cell line SKOV3 was obtained from the Shanghai Cell Bank of Chinese Academy of Sciences (Shanghai, China), and A2780 was from the Shandong Academy of Medical Sciences (Jinan, China). Cells were cultured in RPMI 1640 with 10\% fetal bovine serum (GIBCO; Grand Island, NY, USA) at $37^{\circ} \mathrm{C}, 5 \% \mathrm{CO}_{2}$ and incubated with $80 \mu \mathrm{g} / \mathrm{mL}$ (for SKOV3 cells) or $40 \mu \mathrm{g} / \mathrm{mL}$ (for A2780 cells) of 20 (S)Rg3 for $24 \mathrm{~h}$ or $48 \mathrm{~h}$.

\section{IncRNA deep sequencing and bioinformatics analysis}

Deep sequencing was performed, and the data were analyzed by Novel Bioinformatics Ltd. Co. (Shanghai, China). Total RNAs were extracted from SKOV3 cells treated with 0 or $80 \mu \mathrm{g} / \mathrm{mL} 20(\mathrm{~S})-\operatorname{Rg} 3$ for 24 $\mathrm{h}$ by TRIzol reagent (Invitrogen; Carlsbad, CA, USA), and RNA with RIN>8.0 was utilized to construct rRNA depletion library (VAHTSTM Total RNA-seq (H/M/R)) according to the manufacturer's instructions. Raw data sequenced on the Ion Torrent Proton platform was filtered (removing the adaptor sequences, reads with $>5 \%$ ambiguous bases (noted as $\mathrm{N}$ ) and low-quality reads containing more than 20 percent of bases with qualities of $<20$ ). Clean data was then mapped to Human genome (GRCh38) utilizing HISAT2 algorithm [13]. HTSeq [14] was used to calculate the gene counts of lncRNAs. Differentially expressed lncRNA analysis was performed with DESeq algorithm [15] under following criteria: Fold Change $>2$, p-value $<0.05$ and FDR $<0.05$. The Miranda package [16] was used to predict microRNA target on the full length sequence of differentially expressed lncRNA.

\section{Quantitative real-time PCR}

Total RNA was extracted using TRIzol (Invitrogen; Carlsbad, CA, USA) and the concentrations were quantified on a UV spectrophotometer (Bio-Rad Inc.; Hercules, CA, USA). cDNA was synthesized according to the manufacturer's instructions of PrimeScript ${ }^{\mathrm{TM}}$ RT reagent kit with gDNA Eraser (TaKaRa; Dalian, China). Quantitative real-time PCR was performed on a CFX 96 real-time PCR system (Bio-Rad Inc,; Hercules, CA, USA) with SYBR Premix Ex Taq ${ }^{\mathrm{TM}}$ II kit (TaKaRa; Dalian, China). $\beta$-actin was used as internal control of IncRNA and mRNA, and U6 was the internal control of microRNA. Relative gene expression was calculated according to $2^{-\Delta \Delta C \mathrm{t}}$ method. The oligonucleotide primers were synthesized by BGI (Shenzhen, China) and microRNA primers were synthesized by RiboBio Co. Ltd. (Guangzhou, China). Sequences of primers were as follows: H19 F 5'-TGC TGC ACT TTA CAA CCA CTG-3', R 5'-ATG GTG TCT TTG ATG TTG GGC-3'; PKM2 F 5'-TCC GGA TCT CTT CGT CTT TG-3', R 5'-GTC TGA ATG AAG GCA GTC CC-3'; $\beta$-actin F 5'-TCC CTG GAG AAG AGC TAC GA-3', R 5'-AGC ACT GTG TTG GCG TAC AG-3'.

\section{Western blotting}

Total protein was extracted using RIPA buffer containing protease inhibitors (Roche; Indianapolis, IN, USA). Protein concentrations were quantified with the BCA-200 Protein Assay kit (Pierce; Rockford, IL, USA). Proteins were separated on 10\% SDS-polyacrylamide gels and transferred onto nitrocellulose membranes (Pall Life Science; Port Washington, NY, USA). Then the membranes were blocked in 5\% non-fat milk at room temperature for 1 hour and incubated at $4^{\circ} \mathrm{C}$ overnight with the primary antibodies (PKM2 1:10000, $\beta$-actin 1:1000, Ago2 1:500). After washing with 0.5\% TBST, the membranes were incubated with HRP-conjugated secondary antibodies, and the blots were developed using ECL reagents (Millipore; Billerica, MA, USA) by a chemiluminescence imaging system (Bio-Rad Inc., Hercules, CA, USA).

\section{Glucose consumption and lactate production assay}

The supernatant of cell culture media was collected to detect the levels of glucose and lactate separately with Glucose Assay kit and Lactic Acid Assay kit (Nanjing Jiancheng Bioengineering Institute; Nanjing, China) according to the manufacturer's instructions. The optical density at $505 \mathrm{~nm}$ for glucose measurement and $530 \mathrm{~nm}$ for lactate measurement were read on an EnSpire multimode plate reader (PerkinElmer; Waltham, MA, USA). Glucose consumption and lactate production were calculated based on the absorption values and normalized to the cell numbers. 


\section{Cellular Physiology Cell Physiol Biochem 2018;51:1340-1353 \\ \begin{tabular}{ll|l} 
Dol: 2018 The Author(s). Published by S. Karger AG, Basel \\
and Biochemistry
\end{tabular}}

miRNA inhibitor, miRNA mimic, small interfering RNA and plasmid transient transfection

hsa-miR-324-5p inhibitor and mimic were purchased from RiboBio Co. Ltd. (Guangzhou, China). H19 siRNAs were synthesized by GenePharma (Shanghai, China). Sequences of siRNAs were as follows: siH19a5'-UAA GUC AUU UGC ACU GGU UTT-3'; siH19-b 5'-CCA ACA UCA AAG ACA CCA UTT-3'; negative control siRNA 5'-GCG ACG AUC UGC CUA AGA UTT-3'. The human H19-expressing plasmid was constructed by Genechem (Shanghai, China). Cells were seeded into 6-well plates. hsa-miR-324-5p inhibitor and mimic and H19 siRNAs were transfected at 30-50\% confluency with X-treme GENE siRNA transfection reagent (Roche; Indianapolis, IN, USA) according to the manufacturer's instructions. The H19-expressing plasmid was transfected at 70-90\% confluency with X-treme GENE HP DNA transfection reagent (Roche; Indianapolis, IN, USA). After $48 \mathrm{~h}$ or $72 \mathrm{~h}$ incubation, cells were harvested for further study.

\section{Plasmid construction and dual-luciferase reporter assay}

The human H19 gene and the 3'UTR of human PKM2 gene containing predicted miR-324-5p binding sites were amplified from genomic DNA of SKOV3 using the following primers:

H19 F 5'-CCC AAG CTT GGG CAG CCA CCA CAT CAT CCC AG-3', R 5'-GGA CTA GTC CCA GAG TCG TGG AGG CTT TGA-3';

PKM2 3'UTR F 5'-CCC AAG CTT GGG GGA GGA ATG CTG GAC TGG AG-3', R 5'-CCC AAG CTT GGG GGA GGA ATG CTG GAC TGG AG-3'.

The PCR products were recovered from running gels, digested with Hind III and Spe I endonuclease and inserted into pMIR-REPORT luciferase vector (Ambion; Austin, TX, USA) to construct H19-WT vector and PKM2-WT 3'UTR vector. The transversion mutants in the miR-324-5p binding sites were generated by overlap extension method to construct H19-MUT vector and PKM2-MUT 3'UTR vector. The primers were used as follows:

H19-MUT P1: 5'-CCCAAGCTTGGGCAGCCACCACATCATCCCAG-3',

P2: 5'-AGCTCCTCCAGCCTTCATACGCCGTCCCCACCA-3',

P3: 5'-TGGTGGGGACGGCGTATGAAGGCTGGAGGAGCT-3',

P4: 5'-GGACTAGTCCCAGAGTCGTGGAGGCTTTGA-3';

PKM2-MUT 3'UTR P1: 5'-CCCAAGCTTGGGGGAGGAATGCTGGACTGGAG-3',

P2: 5'-CCTTTAGAAAAAATCCTACGCCAGAGGACTCCC-3',

P3: 5'-GGGAGTCCTCTGGCGTAGGATTTTTTCTAAAGG-3',

P4: 5'-GGACTAGTCCTGGCTGTTTCTTGACCCCAA-3'.

All the recombinant vectors were confirmed by sequencing. Cells were seeded into 24 -well plates. pRL-TK vectors $(20 \mathrm{ng})$ and wild-type or mutation vectors $(180 \mathrm{ng}$ ) were co-transfected along with $20 \mathrm{nM}$ of miR324-5p mimic or negative control at 80-90\% confluency with X-treme GENE siRNA transfection reagent (Roche; Indianapolis, IN, USA) according to the manufacturer's instructions. $24 \mathrm{~h}$ after transfection, cells were harvested and the relative firefly luciferase activity (normalized to Renilla luciferase activity) was measured with Dual-Luciferase Reporter Assay System (Promega; Madison, WI, USA) according to the manufacturer's instructions.

\section{RNA-binding protein immunoprecipitation}

Cells were scraped off culture dishes and lysed using complete RIP lysis buffer at 80-90\% confluency using Magna RIP kit (Millipore; Billerica, MA, USA). $100 \mu \mathrm{l}$ of cell lysate was incubated with RIP immunoprecipitation buffer containing magnetic beads conjugated with anti-Ago2 antibody or normal mouse IgG at $4^{\circ} \mathrm{C}$ overnight. The immunoprecipitated RNA was purified by digesting protein with proteinase $\mathrm{K}$ and analyzed by qRT-PCR.

\section{Lentivirus infection}

hsa-miR-324-5p-up lentivirus was constructed by Genechem (Shanghai, China). The lentivirus infected cells with MOI 10 at $20-30 \%$ confluency. The cells were then used for cell viability assay, colony formation assay and xenograft tumor model. 


\section{Cellular Physiology Cell Physiol Biochem 2018;51:1340-1353

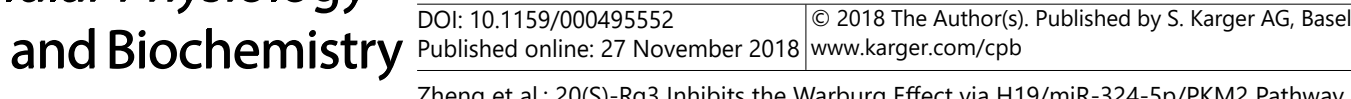

Cell viability assay

1200 cells/well were seeded into 96-well plates. Cell viability was examined with Cell Counting Kit-8 ( 7 seapharmtech Co. Ltd.; Shanghai, China) for 7 days. $10 \mu \mathrm{l}$ of CCK-8 was added into $100 \mu \mathrm{l}$ of culture media, and cells were incubated at $37^{\circ} \mathrm{C}$ for $0.5 \mathrm{~h}$. The $450 \mathrm{~nm}$ wave-length absorption values were measured on an EnSpire multimode plate reader (PerkinElmer; Waltham, MA, USA). The detections were performed in triplicate wells on the same plate in three independent experiments.

\section{Colony formation assay}

500 cells/well were seeded into 6-well plates and cultured for 10 days. Cells were fixed with methanol and stained with crystal violet. The images were captured and the numbers of colonies in which the number of cells was more than 50 were counted under microscope.

\section{Xenograft tumor model}

All animal experimental procedures were approved by the Ethics Committee of the First Affiliated Hospital, Xi'an Jiaotong University. Four-week old BALB/c female mice were randomly divided into 2 groups (miR-324-5p and negative control, $n=7$ ) and injected subcutaneously with $2 \times 10^{6}$ cells $(100 \mu \mathrm{l})$ into the right axilla. Mice weight and the length (L) and width (W) of xenograft tumors were measured every 3 days. Xenograft volume $(\mathrm{V})$ was calculated as $\mathrm{V}=\left(\mathrm{L} \times \mathrm{W}^{2}\right) / 2$. The tumors were collected, weighed, and preserved in liquid nitrogen or formalin for further study at day 24 .

\section{Immunohistochemistry staining}

Histologic slides were prepared from the paraffin-embedded subcutaneous xenograft tumor tissue blocks, and then dewaxed in xylene and rehydrated in a descending alcohol series. The specimens were heated in $0.01 \mathrm{M}$ citrate buffer ( $\mathrm{pH} 6.0)$ at $98^{\circ} \mathrm{C}$ for 20 minutes in a microwave oven to retrieve antigenic binding sites and incubated with anti-PKM2 antibody $(1: 800)$ at $4^{\circ} \mathrm{C}$ overnight. After incubation with HRPconjugated secondary antibody at room temperature for 15 minutes, color was developed with DAB. Slides were counterstained with hematoxylin, dehydrated in an ascending alcohol series and mounted for analysis. Images were captured with an Olympus BH-2 microscope (Tokyo, Japan) installed with the DeltaPix Camera and software (Maalov, Denmark).

\section{Statistical analysis}

All data were represented as mean \pm standard deviation and analyzed with SPSS software (Chicago, IL, USA). Statistical differences were determined by two-tailed t-test and considered significant $\left(^{*}\right)$ when $\mathrm{P}<0.05$.

\section{Results}

\section{Differential expression of IncRNAs in the 20(S)-Rg3-treated SKOV3 cells}

LncRNA deep sequencing was performed to examine the 20(S)-Rg3-regulated lncRNAs in SKOV3 ovarian cancer cells. The raw lncRNA sequencing data was submitted to Gene Expression Omnibus (GEO) database and accessed with the GEO accession number GSE118216 (https://www.ncbi.nlm.nih.gov/geo/query/acc.cgi?acc=GSE118216). A total of 5305 lncRNAs were detected in 20(S)-Rg3-treated SKOV3 cells and negative control cells, including 1524 ncRNAs and 3781 pseudo genes. Compared to the control cells, 67 lncRNAs were significantly decreased (fold change $<0.5, \mathrm{p}<0.05$ ) including 12 ncRNAs and 55 pseudo genes, and 18 lncRNAs were significantly increased (fold change $>1.5, \mathrm{p}<0.05$ ) including 3 ncRNAs, 14 pseudo genes and 1 unknown gene in the 20(S)-Rg3-treated SKOV3 cells (Fig. 1A). The fold change (FC) and the corresponding false discovery rate (FDR) for the differentially expressed lncRNAs between 20(S)-Rg3-treated and non-treated SKOV3 cells were summarized in Table 1 . Among the significantly differentially expressed lncRNAs, 4 lncRNAs presented the lowest FDR value $(F D R=0)$ including downregulated pseudo gene RNU1-19P and ncRNA H19, and pseudo genes LOC93622 and CICP7. 
Fig. 1. The expression patterns of 85 differentially expressed lncRNAs in RNA-seq.

(A) $\mathrm{H}$ i e $\mathrm{r}$ a $\mathrm{c} \mathrm{h}$ i c a l clustering of 85 d i f f e re n t i a lly expressed lncRNAs in the 20(S)-Rg3treated SKOV3 cells versus negative control cells were displayed in a heatmap (fold change of $>1.5$ or $<0.5$, FDR $<0.05$ ). Each column represented one library, and the color bar indicated the relative expression level from low (green) to high (red). $\quad E X P=20(S)$ Rg3-treated SKOV3 cells. $\mathrm{NC}=$ negative control SKOV3 cells. (B) The expression $\log _{2}$ FC (fold change, $\mathrm{FC}$ ) values in the 20 (S)-Rg 3 -treated SKOV3 cells versus negative control cells detected by RNAseq were calculated by readcount. (C) Relative expression of the H19 was quantified by qRTPCR. Compared with the control group, H19 was significantly decreased in the 20 (S)-Rg3-treated group. $\quad * \mathrm{P}<0.05$, t-test.

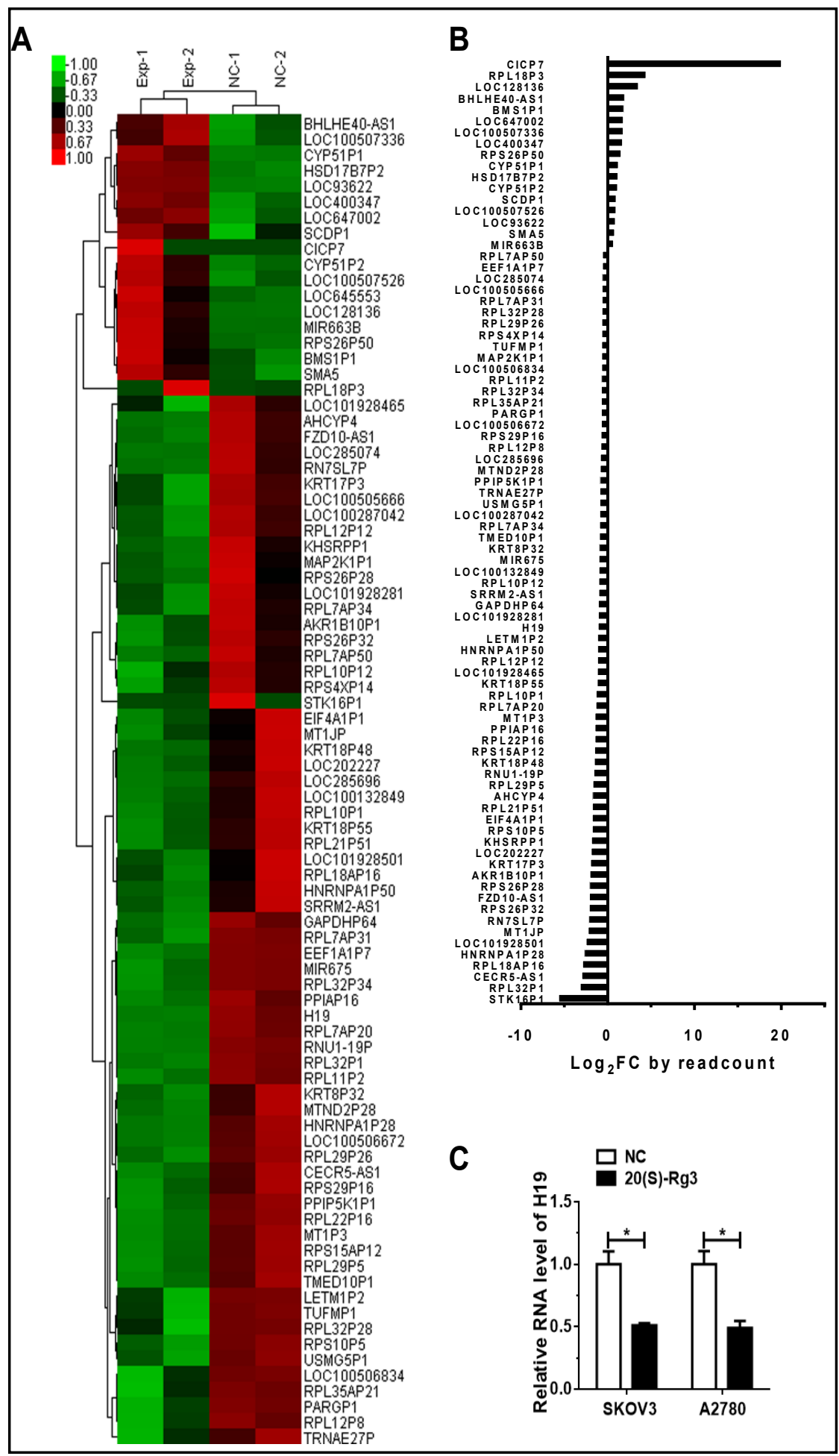

20(S)-Rg3 downregulated H19 to inhibit the Warburg effect in ovarian cancer cells

H19 level was examined in 20(S)-Rg3-treated SKOV3 and A2780 ovarian cancer cells by qRT-PCR. Consistent with IncRNA deep sequencing results (Fig. 1B), H19 was found to be decreased by $50 \%$ in $20(\mathrm{~S})$-Rg3-treated cells compared to control cells (Fig. 1C). The role of $\mathrm{H} 19$ in the inhibition of 20(S)-Rg3 on the Warburg effect was then examined. Knocking down of H19 by siRNAs in SKOV3 and A2780 cells (Fig. 2A) reduced the glucose consumption 


\section{Cellular Physiology Cell Physiol Biochem 2018;51:1340-1353

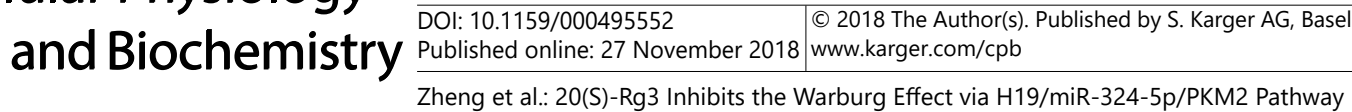

(Fig. 2B) and lactate production (Fig. 2C), and decreased PKM2 rather than HK2 expression (Fig. 2D). Overexpression of $\mathrm{H} 19$ in 20(S)-Rg3-treated SKOV3 and A2780 cells (Fig. 2E) reversed the inhibitory effect of $20(\mathrm{~S})-\mathrm{Rg} 3$ on the glucose consumption (Fig. 2F), lactate production (Fig. 2G) and PKM2 expression (Fig. 2H), but had no effect on HK2 expression. These results indicated that $20(\mathrm{~S})$ Rg3 prevented the Warburg effect via suppressing $\mathrm{H} 19$ expression in ovarian cancer cells.

H19 sponged miR-324-5p to enhance PKM2 expression

We then investigated the mechanism of the stimulation of H19 on PKM2 expression. Since H19 was found to act as the microRNAs sponge, we attempted to identify a PKM2-targeting microRNA sponged by H19. We previously reported that 11 microRNAs were significantly upregulated by 20 (S)-Rg3, including miR-3163, miR-664a-5p, miR-67175p, miR-4329, miR-603, miR-3245p, miR-1283, miR-532-3p, miR33a-3p, miR-519a-5p and miR486-3p. Analysis using RNAHybrid algorithm found a binding site of miR-324-5p in H19 sequence located in 1897-1902nt, and PKM2 was predicted as a potential target of miR-324-5p by microRNA.org database. Knocking down of H19 increased miR-324-5p level (Fig. 3A), while overexpression of H19 abrogated the 20(S)-Rg3-stimulated miR-324-5p (Fig. 3B). But inhibition of miR-324-5p in 20(S)-Rg3-treated cells or overexpression of miR-324-5p did not influence the level of H19 (Fig. 3C and 3D). These results indicated H19 negatively regulated miR-324-5p, but not vice versa.

The direct action of H19 on miR-324-5p was further verified by dual-luciferase reporter assay and RNA-binding protein immunoprecipitation (RIP). Dual-luciferase reporter assay showed that miR-324-5p mimic weakened the luciferase activity of H19 WT luciferase reporter vector containing the wild-type miR-324-5p-binding site in H19 sequence, while it had no effect on that of H19 MUT luciferase reporter vector containing the mutant miR324-5p-binding site (Fig. 3E). RIP for Ago 2 followed by qRT-PCR showed that endogenous H19 and miR-324-5p were specifically enriched in the component pulled down by anti-Ago2 antibody (Fig. 3F), manifesting that $\mathrm{H} 19$ was associated with RNA-induced silencing complex composed of miR-324-5p and Ago2 protein.
Table 1. The top thirty lncRNAs most significantly changed in 20(S)-Rg3-treated SKOV3 cells relative to negative control cells

\begin{tabular}{lcccc}
\hline Accession ID & 20 (S)-Rg3 & NC & Fold change & FDR \\
\hline RNU1-19P & 116.652054 & 347.1274 & 0.336049689 & 0 \\
H19 & 4660.3653 & 9894.069 & 0.471026155 & 0 \\
RPL29P26 & 257.340143 & 408.841 & 0.629438135 & $4.01 \mathrm{E}-09$ \\
RPS15AP12 & 34.9791677 & 98.16263 & 0.356338937 & $7.64 \mathrm{E}-09$ \\
RPL7AP31 & 275.912635 & 426.1764 & 0.64741414 & $3.06 \mathrm{E}-08$ \\
RPL12P12 & 63.5927035 & 141.7483 & 0.448631103 & $1.23 \mathrm{E}-07$ \\
RPL32P1 & 3.74074726 & 33.38363 & 0.112053351 & $1.61 \mathrm{E}-07$ \\
RPL32P34 & 174.994415 & 287.0565 & 0.609616596 & $2.83 \mathrm{E}-07$ \\
RN7SL7P & 164.338415 & 737.2274 & 0.222914142 & $5.11 \mathrm{E}-07$ \\
RPS10P5 & 19.2780015 & 65.55466 & 0.294075232 & $8.24 \mathrm{E}-07$ \\
LOC285074 & 165.23248 & 252.8701 & 0.653428211 & $1.89 \mathrm{E}-06$ \\
MTND2P28 & 191.442355 & 346.2095 & 0.552966815 & $5.12 \mathrm{E}-06$ \\
KRT17P3 & 11.5340185 & 45.02861 & 0.256148693 & $8.6 \mathrm{E}-06$ \\
TMED10P1 & 74.9788573 & 143.4249 & 0.522774159 & $1.28 \mathrm{E}-05$ \\
KRT8P32 & 66.1849204 & 129.1888 & 0.512311555 & $1.4 \mathrm{E}-05$ \\
RPL32P28 & 161.844774 & 252.5958 & 0.640726365 & $1.81 \mathrm{E}-05$ \\
PARGP1 & 111.975977 & 184.9885 & 0.605313134 & $1.91 \mathrm{E}-05$ \\
RPS26P32 & 23.5763935 & 102.3762 & 0.230291674 & $7.47 \mathrm{E}-05$ \\
LOC100506672 & 120.54869 & 199.6582 & 0.603775283 & $9.96 \mathrm{E}-05$ \\
RPL11P2 & 122.72253 & 200.1663 & 0.613102926 & 0.000175 \\
GAPDHP64 & 45.8323209 & 96.0118 & 0.477361339 & 0.000327 \\
RPL21P51 & 13.8144587 & 46.03459 & 0.300088696 & 0.000336 \\
FZD10-AS1 & 7.48149453 & 31.97119 & 0.234007379 & 0.000621 \\
PPIP5K1P1 & 67.4973628 & 122.7634 & 0.549816613 & 0.000652 \\
LOC285696 & 62.2636407 & 109.9736 & 0.566169082 & 0.000719 \\
AHCYP4 & 12.3214839 & 40.85565 & 0.301585783 & 0.000748 \\
RRT18P55 & 36.4967867 & 83.38794 & 0.437674653 & 0.001374 \\
\hline & 17.8423386 & 48.56139 & 0.367418201 & 0.001608 \\
LOC100506834 & 145.125749 & 232.7742 & 0.623461356 & 0.001913 \\
& 2.06723998 & 102.8301 & 0.020103451 & 0.00197 \\
\hline
\end{tabular}




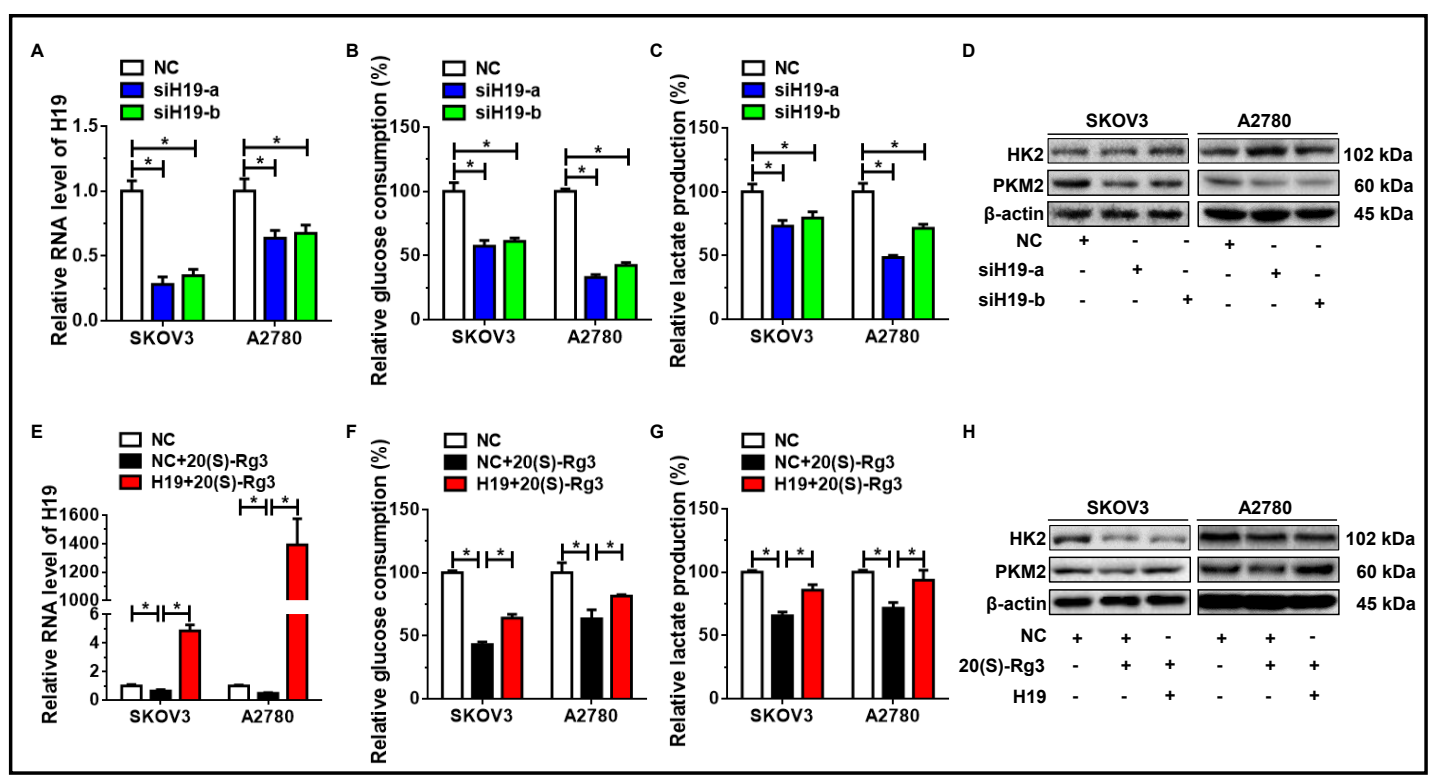

Fig. 2. 20(S)-Rg3 downregulated H19 to antagonize the Warburg effect. (A) H19 was effectively knocked down by two siRNA in both SKOV3 and A2780 ovarian cancer cells after $48 \mathrm{~h}$ transfection. (B) Knocking down of H19 inhibited glucose consumption of SKOV3 and A2780 cells. (C) Knocking down of H19 weakened lactate production in SKOV3 and A2780 cells. (D) Knocking down of H19 reduced PKM2 protein level, while had insignificant effect on HK expression level. (E) H19 was decreased in 20(S)-Rg3-treated cells, and H19 was overexpressed in 20(S)-Rg3-treated cells after transfection of H19-expressing plasmid for $48 \mathrm{~h}$. (F) 20(S)-Rg3 lessened glucose consumption in SKOV3 and A2780 ovarian cancer cells, which was partly recovered by H19 overexpression. (G) H19 overexpression impaired the inhibition of 20(S)-Rg3 on lactate production. (H) H19 overexpression reversed the function of $20(\mathrm{~S})$ - Rg3 on decreasing $\mathrm{PKM} 2$. ${ }^{*} \mathrm{P}<0.05$, $\mathrm{t}$-test.

Next we knocked down both of H19 and miR-324-5p in SKOV3 and A2780 (Fig. 3G). The reduction of glucose consumption and lactate production caused by $\mathrm{H} 19$ interference was prevented by miR-324-5p inhibition (Fig. 3H and 3I). Meanwhile, the protein level of PKM2 in H19 and miR-324-5p co-inhibited cells was increased compared with that in H19inhibited cells (Fig. 3J). These results illustrated that H19 enhanced PKM2 expression to promote the Warburg effect in ovarian cancer cells by sponging miR-324-5p.

\section{miR-324-5p antagonized the Warburg effect via directly inhibiting PKM2}

The effect of miR-324-5p on the Warburg effect in ovarian cancer cells was then examined. Overexpression of miR-324-5p via transfecting miR-324-5p mimic into SKOV3 and A2780 cells (Fig. 4A) reduced the glucose consumption (Fig. 4B) and lactate production (Fig. 4C), and significantly decreased PKM2 protein level (Fig. 4D). Next miR-324-5p was suppressed via transfecting miR-324-5p inhibitor into 20(S)-Rg3-treated SKOV3 and A2780 cells. qRT-PCR results showed that miR-324-5p inhibitor antagonized 20(S)-Rg3-induced elevation of miR-324-5p (Fig. 4E), and reduction of glucose consumption (Fig. 4F), lactate production (Fig. 4G) and PKM2 protein level (Fig. 4H). These results indicated that 20(S)Rg3 inhibited the Warburg effect in ovarian cancer cells by upregulating miR-324-5p.

Online algorithm (microRNA.org) predicted the miR-324-5p-binding site was located in 331-336nt of PKM2 3'UTR. We constructed PKM2 WT-3'UTR containing the miR-324-5pbinding site and PKM2 MUT-3'UTR containing transversion mutations of the miR-324-5pbinding site. Dual-luciferase reporter assay showed that compared with the negative control, miR-324-5p mimics significantly decreased the luciferase activity of PKM2 WT-3'UTR, but had no effect on the luciferase activity of PKM2 MUT-3'UTR (Fig. 4I). These data verified that miR-324-5p directly targeted PKM2. 


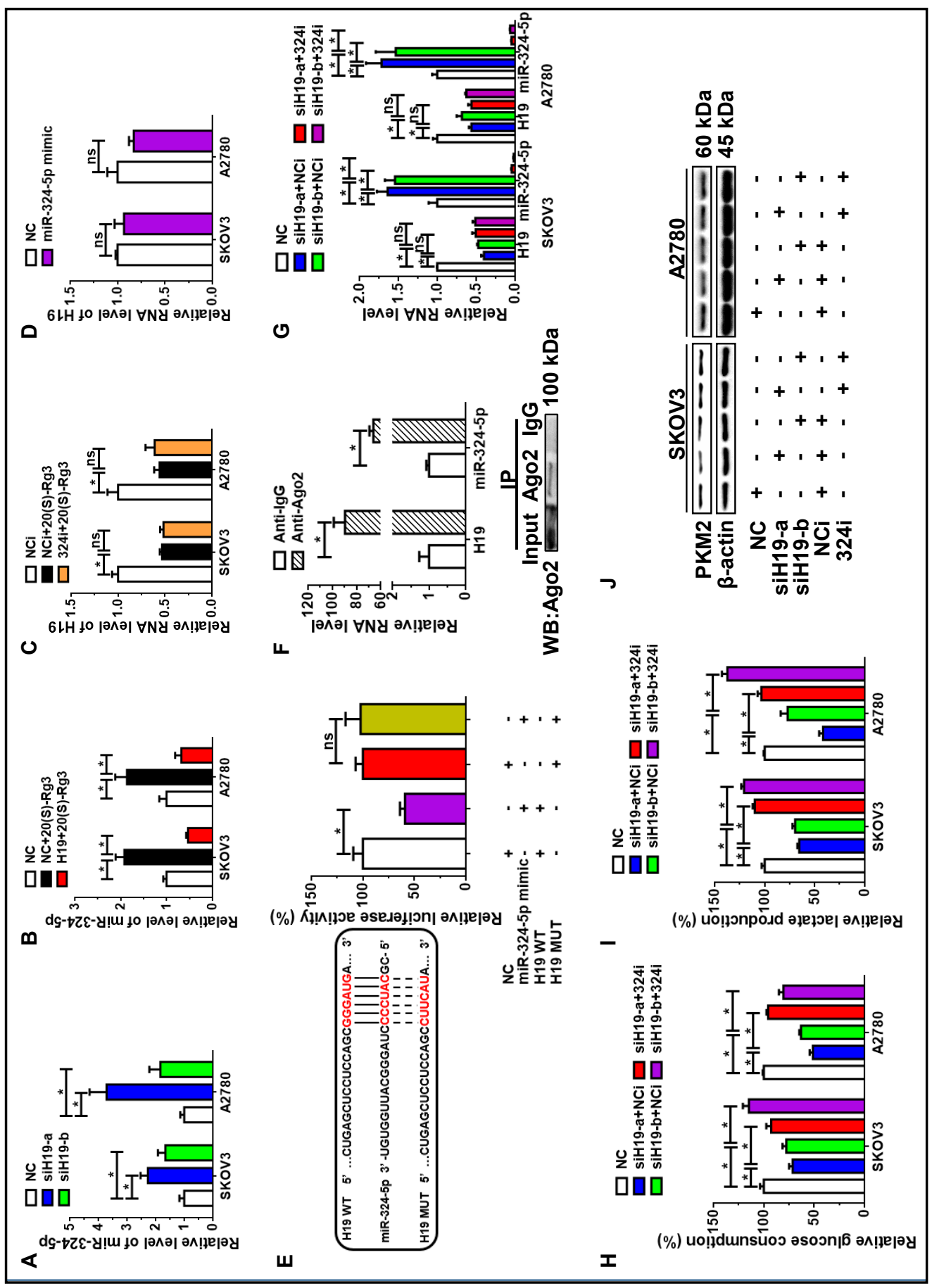

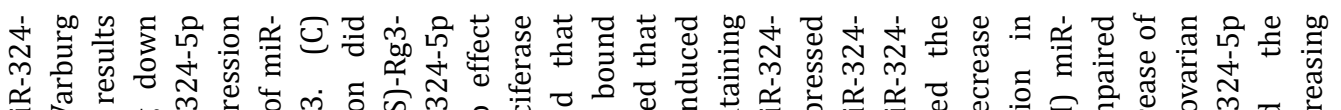

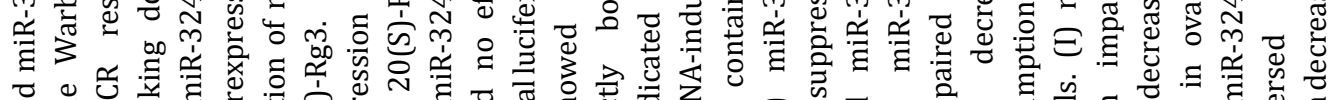

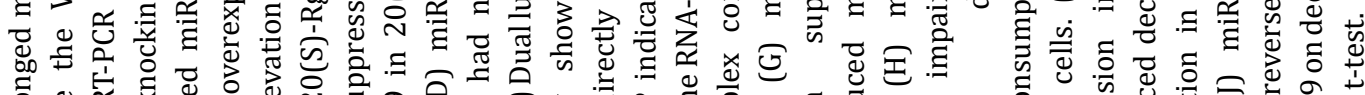

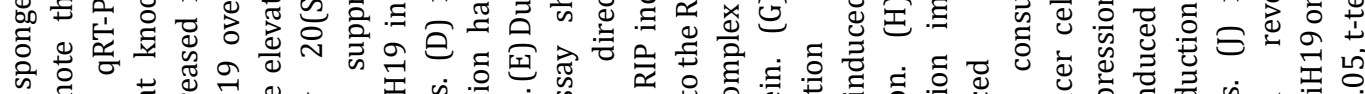
के

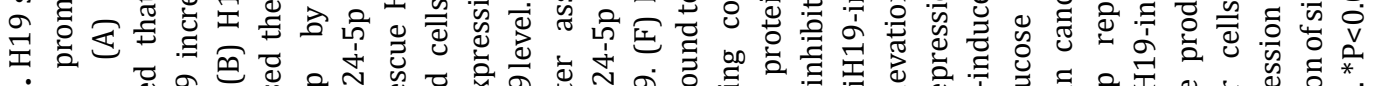

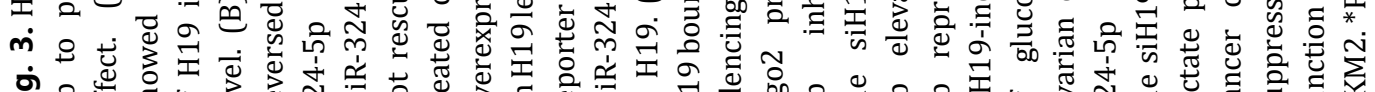

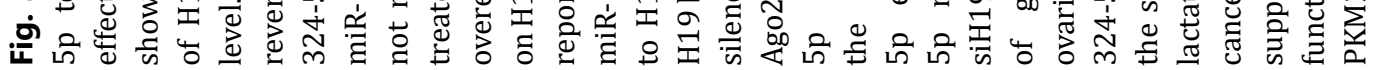




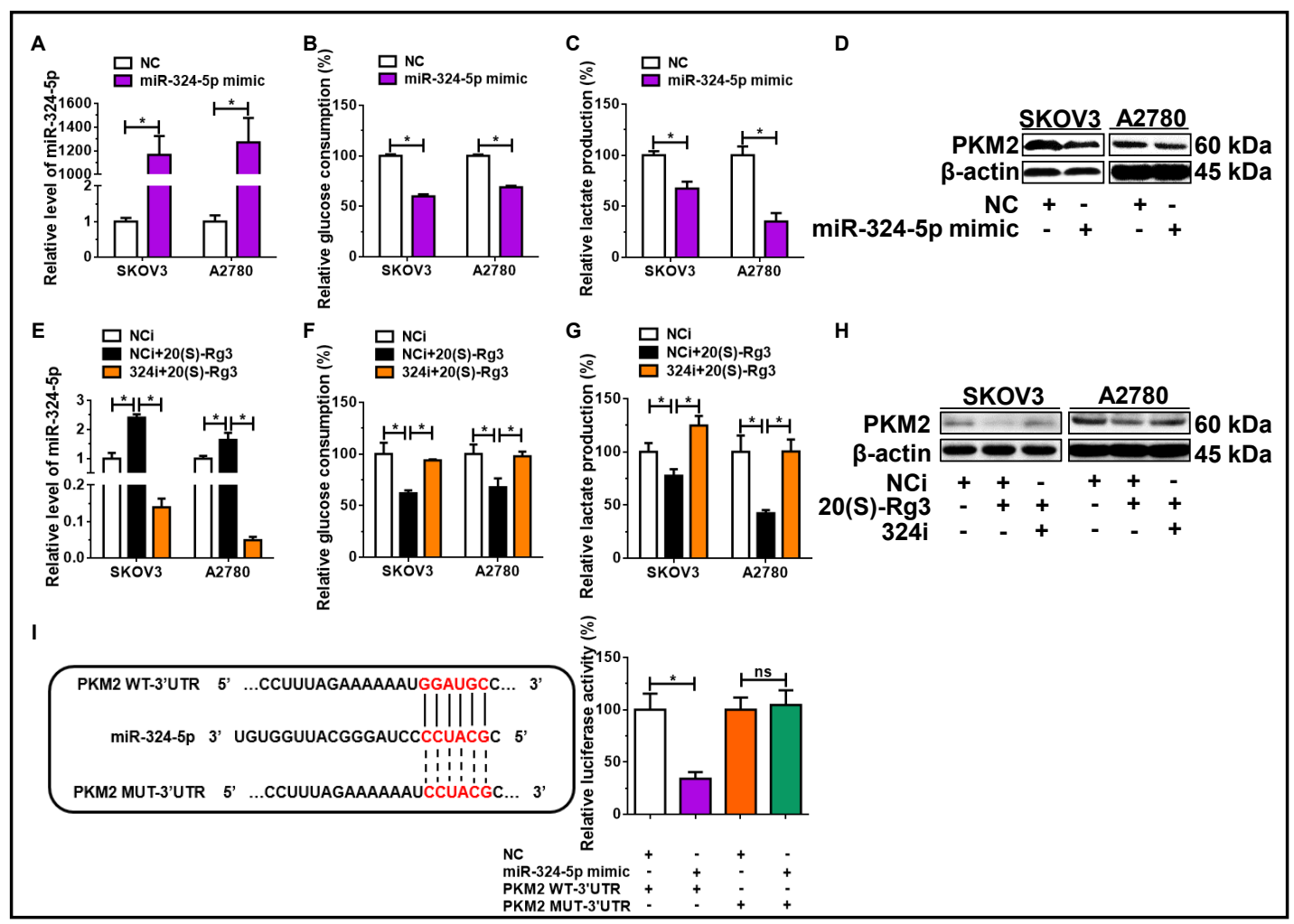

Fig. 4. miR-324-5p targeted PKM2 to antagonize the Warburg effect. (A) qRT-PCR showed that miR-324-5p was overexpressed by miR-324-5p mimic transfection. (B) Glucose consumption was reduced in miR-324$5 p$ overexpressed cells. (C) Lactate production were reduced in miR-324-5p overexpressed cells. (D) PKM2 protein level was decreased in miR-324-5p mimic-transfected ovarian cancer cells. (E) qRT-PCR results showed that miR-324-5p was inhibited by miR-324-5p inhibitor transfection. (F) miR-324-5p suppression impaired the inhibition of $20(\mathrm{~S})-\mathrm{Rg} 3$ on the glucose consumption. (G) miR-324-5p suppression impaired the inhibition of $20(\mathrm{~S})-\mathrm{Rg} 3$ on the lactate production. (H) miR-324-5p suppression reversed the function of 20(S)-Rg3 on decreasing PKM2. (I) Dual-luciferase reporter assay showed that PKM2 was the direct target of miR-324-5p. * $\mathrm{P}<0.05$, t-test.

Overexpression of miR-324-5p inhibited ovarian cancer cell proliferation in vitro and in vivo

To verify the influence of the anti-Warburg effect of miR-324-5p in ovarian cancer cells, SKOV3 cells were infected by hsa-miR-324-5p-up lentivirus to overexpress miR-324-5p (Fig. $5 \mathrm{~A}$ ), and cell proliferation was examined. Compared with the negative control, overexpression of miR-324-5p significantly retarded cell viability (Fig. 5B) and colony formation capability (Fig. 5C), illustrating that miR-324-5p inhibited ovarian cancer cell proliferation in vitro. We then checked the effect of miR-324-5p on the proliferation of ovarian cancer cells in vivo. Nude mice subcutaneous xenograft models were established using miR-324-5p-up SKOV3 cells and negative control cells, respectively. The mice body weights in negative control group and miR-324-5p-up group were comparable (Fig. 5D). But compared with the negative control group, the tumorigenicity of miR-324-5p-up cells was substantially impaired as illustrated by the fewer xenografts and smaller tumor volumes (Fig. 5E and 5F). qRT-PCR results of xenograft tissues showed that miR-324-5p was increased while H19 remained unchanged in miR-324-5p-up group relative to those of the negative control group (Fig. 5G). Immunohistochemistry results showed that PKM2 protein level was decreased in miR-324$5 \mathrm{p}$-up group compared to that in negative control group (Fig. $5 \mathrm{H}$ ). 


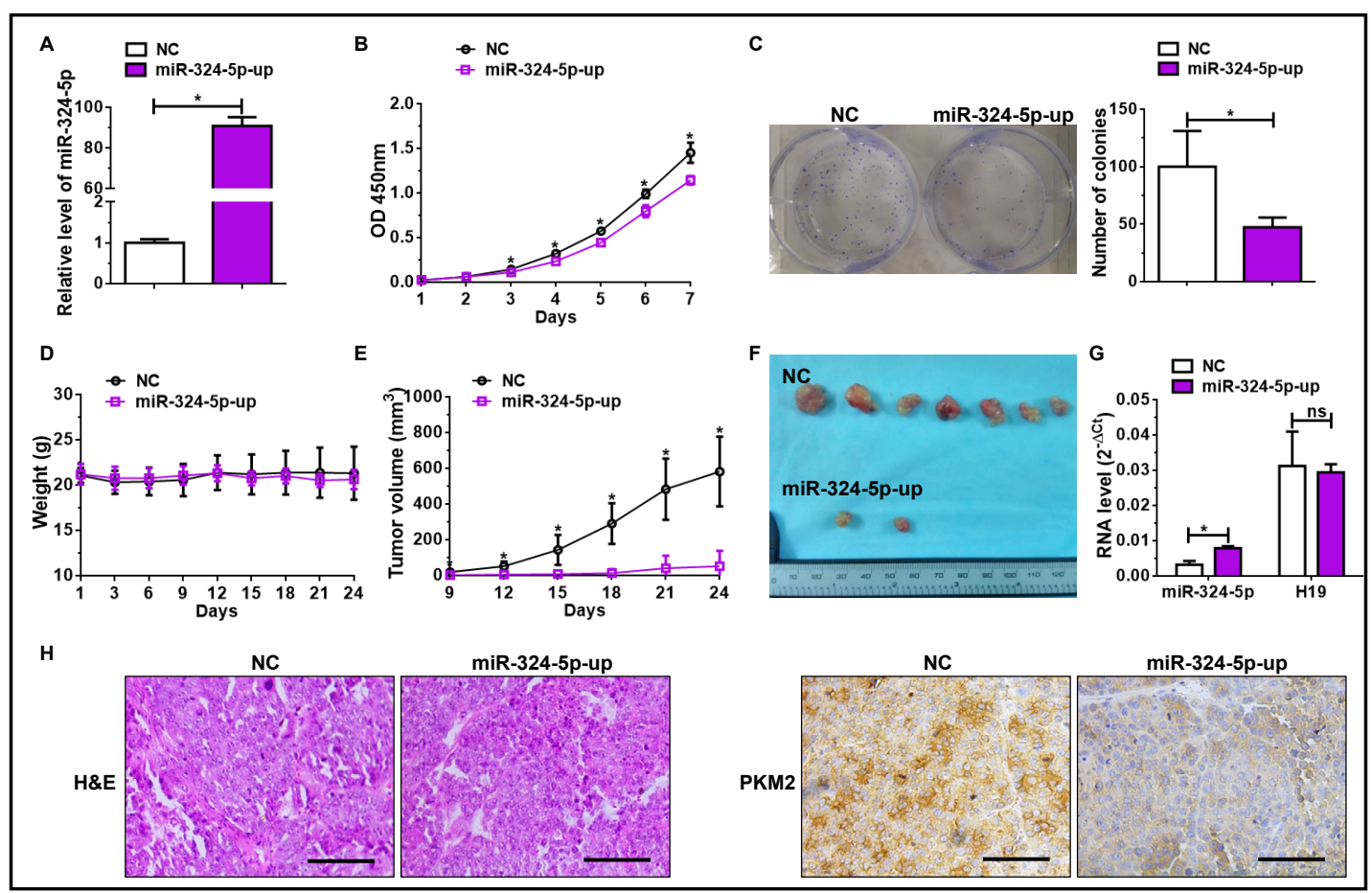

Fig. 5. Overexpression of miR-324-5p inhibited ovarian cancer tumorigenesis in vitro and vivo. (A) miR-324-5p was overexpressed in hsa-miR-324-5p-up lentivirus-infected SKOV3 cells. (B) Cell growth of miR-324-5p overexpressed cells was retarded relative to the negative control cells. (C) miR-324-5p overexpression inhibited cell capability of plate colony formation. (D) The mice weights of miR-324-5pup and control groups were comparable. (E) Tumor volumes of subcutaneous xenografts in miR-324-5pup group were substantially smaller than those of control group. (F) Compared to control group, tumor formation of subcutaneous xenograft in miR-324-5p-up group was significantly inhibited. (G) The level of miR-324-5p was increased, whereas the levels of H19 did not change in miR-324-5p-up group compared with the negative control. $(\mathrm{H})$ Immunohistochemical staining of PKM2 in xenograft tumor tissues (bar scale $50 \mu \mathrm{m}$ ) showed that PKM2 protein level was reduced in miR-324-5p-up group. ${ }^{*} \mathrm{P}<0.05$, t-test.

\section{Discussion}

The Warburg effect is one of the main metabolism ways for ovarian cancer. Research and development of novel therapeutics targeting the Warburg effect will be a feasible strategy to enhance the efficacy of first-line chemotherapeutic drugs and reduce adverse reaction in EOC patients [17]. 20(S)-Rg3 exhibits anti-tumor activity in various cancers via inducing apoptosis or weakening proliferation, migration and invasion of cancer cells [18-20]. Our previous studies have found that 20(S)-Rg3 antagonized the Warburg effect in ovarian cancer and downregulates the two key glycolytic enzymes HK2 and PKM2 [5, 6]. Mechanistic studies have revealed that 20(S)-Rg3 downregulates HK2 via STAT3 [5] or DNMT3A/miR$532-3 p$ [6] pathways. But the mechanism of PKM2 downregulation induced by $20(\mathrm{~S})-\mathrm{Rg} 3$ remains unknown.

We proceeded to investigate the PKM2 downregulation pathway based on previously acquired microRNA sequencing data [6]. PKM2 was predicted and experimentally confirmed as the direct target of 20(S)-Rg3-upregulated miR-324-5p. In view of accumulating evidence proving lncRNAs as microRNA sponges to negatively regulate microRNA function [21-23], we identified the 20(S)-Rg3-influenced IncRNA expression profile with deep sequencing technique to investigate the IncRNA competitively binding miR-324-5p. Fortunately, H19 was screened out from 67 20(S)-Rg3-reduced lncRNAs, with the binding site of miR-324-5p in its sequence. The competitive binding of $\mathrm{H} 19$ to miR-324-5p rescued PKM2 from being

\section{KARGER}




\section{Cellular Physiology Cell Physiol Biochem 2018;51:1340-1353

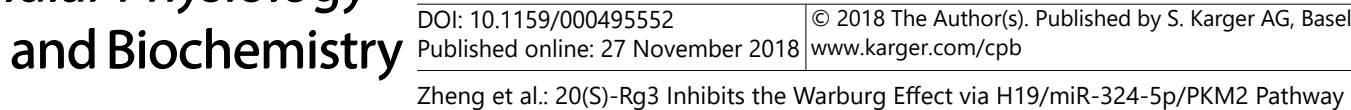

translationally inhibited by miR-324-5p. H19/miR-324-5p/PKM2 pathway was therefore established as the anti-Warburg mechanism of $20(\mathrm{~S})-\mathrm{Rg} 3$.

H19 was overexpressed in various cancers including ovarian cancer, and capable of stimulating cancer cell cycle progression, proliferation, migration and invasion [24, 25]. H19 facilitated tumor development and progression partly via sponging microRNA to recover the expression of microRNA-targeted genes [26-28]. Recently H19 was found to promote glucose metabolism and cell growth in malignant melanoma via competitively binding miR106a-5p to release E2F3 [29]. Here we identified PKM2-targeting miR-324-5p as another target microRNA sponged by H19, and $\mathrm{H} 19$ therefore was a pro-Warburg IncRNA while miR324-5p was an anti-Warburg microRNA. miR-324-5p has been reported as both oncomiR and tumor repressor in cancers because it inhibited cell growth and invasion in multiple myeloma and hepatocellular cancer [30], while promoted proliferation, migration and invasion of papillary thyroid microcarcinoma cells and breast cancer cells [31, 32]. But most published results were from in vitro data. Here, miR-324-5p was verified in vitro and in vivo as a suppressor of ovarian cancer proliferation via negatively regulating the Warburg effect.

In this study, we first reported the 20(S)-Rg3-regulated lncRNA expression profile and identified H19/miR-324-5p/PKM2 pathway responsible for the anti-Warburg activity of 20(S)-Rg3. Further study of 20(S)-Rg3-regulated lncRNA and microRNA could contribute to the understanding of both anti-cancer action of $20(\mathrm{~S})-\mathrm{Rg} 3$ and progression mechanism of ovarian cancer.

\section{Conclusion}

Our results indicated that 20(S)-Rg3 inhibited the Warburg effect via H19/miR-324-5p/ PKM2 pathway to retard growth of ovarian cancer cells.

\section{Acknowledgements}

This work was supported by the National Natural Science Foundation of China (No. 81702576 and No. 30973429), the Natural Science Foundation of Shaanxi Province (No. 2017JM8107) and Foundation of the First Affiliated Hospital of Xi'an Jiaotong University (No. 2016QN-10).

\section{Disclosure Statement}

The authors declare no conflicts of interest.

\section{References}

-1 Siegel RL, Miller KD, Jemal A: Cancer statistics, 2018. CA Cancer J Clin 2018;68:7-30.

- Allemani C, Matsuda T, Di Carlo V, Harewood R, Matz M, Niksic M, Bonaventure A, Valkov M, Johnson CJ, Esteve J, Ogunbiyi OJ, Azevedo ESG, Chen WQ Eser S, Engholm G, Stiller CA, Monnereau A, Woods RR, Visser O, Lim GH et al.: Global surveillance of trends in cancer survival 2000-14 (CONCORD-3): analysis of individual records for 37513025 patients diagnosed with one of 18 cancers from 322 population-based registries in 71 countries. Lancet 2018;10.1016/S0140-6736(17)33326-3.

-3 Kroeger PT, Jr., Drapkin R: Pathogenesis and heterogeneity of ovarian cancer. Curr Opin Obstet Gynecol 2017;29:26-34.

4 Nag SA, Qin JJ, Wang W, Wang MH, Wang H, Zhang R: Ginsenosides as Anticancer Agents: In vitro and in vivo Activities, Structure-Activity Relationships, and Molecular Mechanisms of Action. Front Pharmacol 2012;3:25. 


\section{Cellular Physiology Cell Physiol Biochem 2018;51:1340-1353 \begin{tabular}{ll|l} 
and Biochemistry & $\begin{array}{l}\text { DOl: 10.1159/000495552 } \\
\text { Published online:2 } 27 \text { November } 2018\end{array}$ & $\begin{array}{l}\text { O 2018 The Author(s). Published by S. Karger AG, Basel } \\
\text { www.karger.com/cpb }\end{array}$ \\
\cline { 2 - 3 }
\end{tabular}

5 Li J, Liu T, Zhao L, Chen W, Hou H, Ye Z, Li X: Ginsenoside 20(S)Rg3 inhibits the Warburg effect through STAT3 pathways in ovarian cancer cells. Int J Oncol 2015;46:775-781.

6 Zhou Y, Zheng X, Lu J, Chen W, Li X, Zhao L: Ginsenoside 20(S)-Rg3 Inhibits the Warburg Effect Via Modulating DNMT3A/ MiR-532-3p/HK2 Pathway in Ovarian Cancer Cells. Cell Physiol Biochem 2018;45:2548-2559.

7 Pavlova NN, Thompson CB: The Emerging Hallmarks of Cancer Metabolism. Cell Metab 2016;23:27-47.

$>8$ Yu L, Chen X, Sun X, Wang L, Chen S: The Glycolytic Switch in Tumors: How Many Players Are Involved? J Cancer 2017;8:3430-3440.

-9 Xiang S, Gu H, Jin L, Thorne RF, Zhang XD, Wu M: LncRNA IDH1-AS1 links the functions of c-Myc and HIF1alpha via IDH1 to regulate the Warburg effect. Proc Natl Acad Sci U S A 2018;115:E1465-E1474.

10 Li Z, Li X, Wu S, Xue M, Chen W: Long non-coding RNA UCA1 promotes glycolysis by upregulating hexokinase 2 through the mTOR-STAT3/microRNA143 pathway. Cancer Sci 2014;105:951-955.

11 Ye H, Zhou Q Zheng S, Li G, Lin Q, Ye L, Wang Y, Wei L, Zhao X, Li W, Fu Z, Liu Y, Li Z, Chen R: FEZF1-AS1/ miR-107/ZNF312B axis facilitates progression and Warburg effect in pancreatic ductal adenocarcinoma. Cell Death Dis 2018;9:34.

12 Song J, Wu X, Liu F, Li M, Sun Y, Wang Y, Wang C, Zhu K, Jia X, Wang B, Ma X: Long non-coding RNA PVT1 promotes glycolysis and tumor progression by regulating miR-497/HK2 axis in osteosarcoma. Biochem Biophys Res Commun 2017;490:217-224.

13 Kim D, Langmead B, Salzberg SL: HISAT: a fast spliced aligner with low memory requirements. Nat Methods 2015;12:357-360.

14 Anders S, Pyl PT, Huber W: HTSeq--a Python framework to work with high-throughput sequencing data. Bioinformatics 2015;31:166-169.

-15 Anders S, Huber W: Differential expression analysis for sequence count data. Genome Biol 2010;11:R106.

-16 Enright AJ, John B, Gaul U, Tuschl T, Sander C, Marks DS: MicroRNA targets in Drosophila. Genome Biol 2003;5:R1.

17 Suh DH, Kim HS, Kim B, Song YS: Metabolic orchestration between cancer cells and tumor microenvironment as a co-evolutionary source of chemoresistance in ovarian cancer: a therapeutic implication. Biochem Pharmacol 2014;92:43-54.

18 Lee JY, Jung KH, Morgan MJ, Kang YR, Lee HS, Koo GB, Hong SS, Kwon SW, Kim YS: Sensitization of TRAIL-induced cell death by 20(S)-ginsenoside Rg3 via CHOP-mediated DR5 upregulation in human hepatocellular carcinoma cells. Mol Cancer Ther 2013;12:274-285.

19 Li J, Lu J, Ye Z, Han X, Zheng X, Hou H, Chen W, Li X, Zhao L: 20(S)-Rg3 blocked epithelial-mesenchymal transition through DNMT3A/miR-145/FSCN1 in ovarian cancer. Oncotarget 2017;8:53375-53386.

20 Yuan HD, Quan HY, Zhang Y, Kim SH, Chung SH: 20(S)-Ginsenoside Rg3-induced apoptosis in HT-29 colon cancer cells is associated with AMPK signaling pathway. Mol Med Rep 2010;3:825-831.

21 Salmena L, Poliseno L, Tay Y, Kats L, Pandolfi PP: A ceRNA hypothesis: the Rosetta Stone of a hidden RNA language? Cell 2011;146:353-358.

-22 Tay Y, Rinn J, Pandolfi PP: The multilayered complexity of ceRNA crosstalk and competition. Nature 2014;505:344-352.

-23 Thomson DW, Dinger ME: Endogenous microRNA sponges: evidence and controversy. Nat Rev Genet 2016;17:272-283.

24 Medrzycki M, Zhang Y, Zhang W, Cao K, Pan C, Lailler N, McDonald JF, Bouhassira EE, Fan Y: Histone h1.3 suppresses h19 noncoding RNA expression and cell growth of ovarian cancer cells. Cancer Res 2014;74:6463-6473.

25 Zhong Y, Gao D, He S, Shuai C, Peng S: Dysregulated Expression of Long Noncoding RNAs in Ovarian Cancer. Int J Gynecol Cancer 2016;26:1564-1570.

26 Lv M, Zhong Z, Huang M, Tian Q Jiang R, Chen J: IncRNA H19 regulates epithelial-mesenchymal transition and metastasis of bladder cancer by miR-29b-3p as competing endogenous RNA. Biochim Biophys Acta 2017;1864:1887-1899.

27 Li Z, Li Y, Ren K, Li X, Han X, Wang J: Long non-coding RNA H19 promotes the proliferation and invasion of breast cancer through upregulating DNMT1 expression by sponging miR-152. J Biochem Mol Toxicol 2017;31. doi:10.1002/jbt.21933. 


\section{Cellular Physiology Cell Physiol Biochem 2018;51:1340-1353

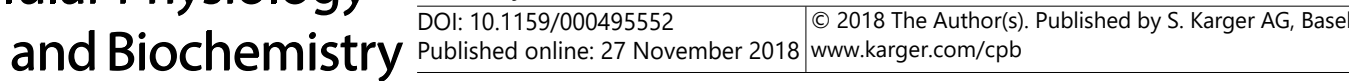

28 Zhou W, Ye XL, Xu J, Cao MG, Fang ZY, Li LY, Guan GH, Liu Q, Qian YH, Xie D: The IncRNA H19 mediates breast cancer cell plasticity during EMT and MET plasticity by differentially sponging miR-200b/c and let-7b. Sci Signal 2017;10:pii:eaak9557.

29 Luan W, Zhou Z, Ni X, Xia Y, Wang J, Yan Y, Xu B: Long non-coding RNA H19 promotes glucose metabolism and cell growth in malignant melanoma via miR-106a-5p/E2F3 axis. J Cancer Res Clin Oncol 2018;144:531-542.

-30 Tang B, Xu A, Xu J, Huang H, Chen L, Su Y, Zhang L, Li J, Fan F, Deng J, Tang L, Sun C, Hu Y: MicroRNA-3245 p regulates stemness, pathogenesis and sensitivity to bortezomib in multiple myeloma cells by targeting hedgehog signaling. Int J Cancer 2018;142:109-120.

-31 Yang Y, Xia S, Ni X, Ni Z, Zhang L, Wang W, Kong Y, Wang Y, Ye L, Zhan W: MiR-324-5p assists ultrasonography in predicting lymph node metastasis of unifocal papillary thyroid microcarcinoma without extracapsular spread. Oncotarget 2017;8:83802-83816.

-32 Song L, Liu D, Zhao Y, He J, Kang H, Dai Z, Wang X, Zhang S, Zan Y: Sinomenine inhibits breast cancer cell invasion and migration by suppressing NF-kappaB activation mediated by IL-4/miR-324-5p/CUEDC2 axis. Biochem Biophys Res Commun 2015;464:705-710. 